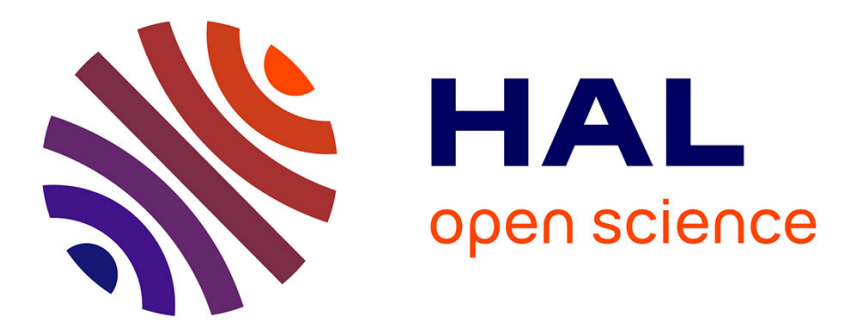

\title{
A promising way to model cracks in composite using Discrete Element Method
}

\author{
Laurent Maheo, Frédéric Dau, Damien Andre, Jean-Luc Charles, Ivan
}

Iordanoff

\section{- To cite this version:}

Laurent Maheo, Frédéric Dau, Damien Andre, Jean-Luc Charles, Ivan Iordanoff. A promising way to model cracks in composite using Discrete Element Method. Composites Part B: Engineering, 2015, 71, pp.193-202. 10.1016/j.compositesb.2014.11.032 . hal-01122922

\section{HAL Id: hal-01122922 \\ https://hal.science/hal-01122922}

Submitted on 4 Mar 2015

HAL is a multi-disciplinary open access archive for the deposit and dissemination of scientific research documents, whether they are published or not. The documents may come from teaching and research institutions in France or abroad, or from public or private research centers.
L'archive ouverte pluridisciplinaire $\mathbf{H A L}$, est destinée au dépôt et à la diffusion de documents scientifiques de niveau recherche, publiés ou non, émanant des établissements d'enseignement et de recherche français ou étrangers, des laboratoires publics ou privés. 


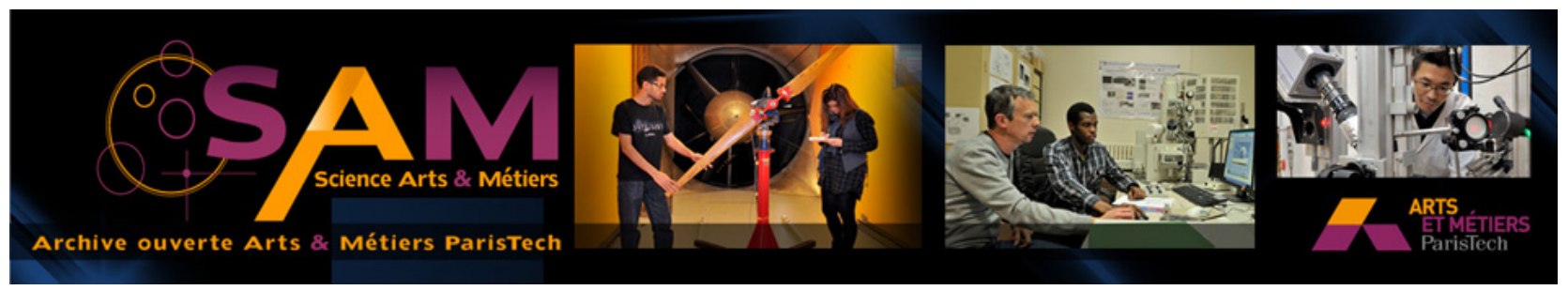

Science Arts \& Métiers (SAM)

is an open access repository that collects the work of Arts et Métiers ParisTech researchers and makes it freely available over the web where possible.

This is an author-deposited version published in: http://sam.ensam.eu

Handle ID: .http://hdl.handle.net/10985/9374

\section{To cite this version :}

Laurent MAHEO, Frédéric DAU, Damien ANDRE, Jean-Luc CHARLES, Ivan IORDANOFF - A promising way to model cracks in composite using Discrete Element Method - Composites Part B: Engineering - Vol. 71, p.193-202 - 2015 


\title{
A promising way to model cracks in composite using Discrete Element Method
}

\author{
L. Maheo ${ }^{\mathrm{a}, \mathrm{b}, *}, \mathrm{~F}$. Dau $^{\mathrm{c}}$, D. André ${ }^{\mathrm{c}}$, J.L. Charles ${ }^{\mathrm{c}}$, I. Iordanoff ${ }^{\mathrm{c}}$ \\ ${ }^{a}$ Univ. Bretagne Sud, LIMATB, F-56100 Lorient, France \\ ${ }^{\mathrm{b}}$ Ecoles Saint-Cyr Coetquidan, CREC, F-56380 Guer, France \\ ${ }^{\text {c } A r t s ~ e t ~ M e t i e r s ~ P a r i s T e c h, ~ l a b o r a t o i r e ~ I 2 M, ~ d p t . ~ D u M A S, ~ U M R ~ C N R S ~ 5295, ~ F-33405 ~ T a l e n c e, ~ F r a n c e ~}$
}

Keywords:

B. Fracture

B. Mechanical properties

C. Computational modeling

C. Damage mechanics

Discrete Element Method

\begin{abstract}
A B S T R A C T
In this article, the Discrete Element Method (DEM) is taking advantage for the damage modeling of a composite material. At this stage of work, a Representative Elementary Volume (REV) of an unidirectional composite material modeled in 3D is considered to prove the relevance of the approach. The interest to introduce the Discrete Elements (DE) on the scale of constituents (fiber and matrix) is to be able to report local mechanisms of degradation such as the matrix micro-fissuring, the fiber/matrix debonding and the break of fiber, appropriate to this type of material. The short-term objective is to use this DEM modeling to treat locally the damages induced by an impact loading associated with a conventional Finite Element modeling beyond the damaged zone. First, the geometrical modelings of the fiber and the matrix are presented. The phase of calibration of the DE model intrinsic parameters governing the fiber and matrix behavior and the fiber/matrix interface is afterward retailed. At this stage, each constituent is assumed to be brittle elastic. Then, simulations of longitudinal and transversal tensions but also of in plane and out of plane shearing are performed on the REV using DEM. The results are discussed and compared with those known for the literature. The capacity of the present DEM to capture the crack paths is particularly highlighted.
\end{abstract}

\section{Introduction}

The increasing market of composite in the aeronautical sector in particular imposes statutory requirements for the safety of the properties and the persons. Concerning the composite material, a major industrial stake is to propose a structural material performing against impacts such as falls of tools during the maintenance, tire debris projections or hail storm. Faced with the need to strongly reduce experiments for the benefit of numerical simulations, the issue is then to develop digital models always more efficient. Then, the trend is to favor multiscale approaches allowing a dialogue between a local damaged zone and the global behavior of the considered structure. Some authors consider the multiscale approach by global/local iterative calculations performed on the whole structure (macroscopic scale) and more locally around the process zones (microscopic scale). The most common methods consist of returning the structures effects (macro scale) to a fine model (micro scale) using adaptive boundary conditions (down-

* Corresponding author at: Ecoles Saint-Cyr Coetquidan, CREC, F-56381 Guer, France. Tel.: +33290404049.

E-mail address: laurent.maheo@univ-ubs.fr (L. Maheo). ward methods) [1,2] and reinjecting a corrective load (multi-grid and decomposition methods) [3,4] or degraded homogenized properties [5] from the local scale to the global scale. Others authors propose a multiscale method for micro-macro failure of composites computing an equivalent discontinuity at macro scale [6].

Others attempt to finalize modelings in which models intended to report at the same time local and global effects using coupling techniques (sticking methods). Within this framework, a direct coupling approach is proposed by the authors of the present study. It consists of introducing locally the Discrete Elements Method, DEM, initially developed by [7] and adapted to the degradation of a continuous medium when cracking or fragmentations appear [8] and coupling it with a more conventional continuous method beyond the process zone. So, the works [9] presents the Discrete Elements Method (DEM) coupled with the Constrained Natural Element Method (CNEM) using Arlequin technique[10]. More recently, this coupling was taken advantage to treat the case of a laser impact on glass [11]. In order to generally deal with the case of impact on composite target, the originality of this work is to propose a 3D modeling using DE for a composite medium. The case of an UD cell is considered at the moment but an extension to UD ply and composite textile is also planned. 
Until now, the use of DEM to model composites is almost restricted to a $2 \mathrm{D}$ domain with studies on the damage observed in a fold UD [12-14] during debonding tests [15-17]. The DEM turns out to be an excellent tool to identify qualitatively the initiation and the distribution of fiber/matrix interfacial debonding [16] and to quantitatively determine the degraded mechanical properties; the degradation of the Young modulus can be related to the density of cracks inside the material [12]. Thanks to growing computing powers, works on the use of the DEM in a 3D domain for homogeneous materials as silica [18] or heterogeneous as the concrete [19] is now possible. Shiu et al. [19] shows in particular the capabilities of the method to predict the depth of penetration of missiles according to their shape. More recently, Aghazadeh Mohandesi et al. [20] used the DEM to prove its capability to describe the behavior of a sand PET composite under compressive loadings and with different temperature conditions.

Our objective is thus to introduce the DEM developed in the laboratory [21] to prove its capability and its relevance to treat the cracks propagation at the microscopic scale. The case of an UD is considered in this work through a Representative Elementary Volume (REV) constituted by a fiber flooded in a cube of resin. The geometrical modeling of the fiber and the matrix is first presented). The filling technique is particularly described. The mechanical modeling of the continuous media constituted by the fiber and the matrix is then handled. It consists of the introduction and calibration of mechanical links to report a brittle elastic behavior. Cohesive beams developed in $[26,27]$ stand for these links. The calibration step to identify beam parameters at microscopic scale is detailed for each constituent (fiber and matrix) and for the interface (fiber/matrix). Numerical tests to validate the REV behavior are then presented. Longitudinal and transversal tractions, and in plane and out of plane shearing are performed on the REV. The parametric simulations qualitatively and quantitatively show the relevance of the present DE model.

Comparisons are also made between two criteria implemented for the failure process. Some conclusions and perspectives conclude this work.

\section{Geometrical modeling of the heterogeneous media}

\subsection{General considerations}

The geometrical modeling of the fiber and the matrix is guided by the following requirements and assumptions: (i) use the simplest DE shape, (ii) adopt a compatible DE size with the scale of mechanisms to observe, (iii) adopt a radius distribution to get a correct representation of the continuous medium (compaction and isotropy), and (iv) use a sufficient number of DE to ensure the macroscopic results non sensitivity to the discretization.

In the present DEM, the formulation is naturally in explicit dynamics. The DE geometry associated with the density carries the kinetic information whereas the links connecting the centers of adjacent DE pilot the behavior, see Section 3.1. For efficiency reasons, the implementation and treatment are performed using spherical DE. More complex geometries could be however envisaged by the use of Voronoï cells [22,23]. The size of spheres is varying according to a Gaussian distribution. It is chosen in such a way to be able to analyze the mechanisms of degradation at the scale they occur; the matrix micro-cracking, the fiber/matrix debonding and the failure of fibers are the interesting mechanisms. Their number and their size have also to allow a good geometrical representation of the fiber/matrix interface.

Practically, building the continuous medium (fiber or matrix) consists of placing at one time a set of DE whose radius has been beforehand chosen according to the required distribution. This stuffing operation is followed by a phase of relaxation to get the best cohesion of the isotropic continuous. The last is governed by two criteria: an optimal rate of compaction (ratio between the volume of spherical DE and the enveloping volume) of 6.3 and a minimal number of coordination (number of contacts by DE) of 6 [26].

Even if the objective at this stage is not to study the degradation of the fiber, its modeling uses the same distribution (casting) of DE as the matrix. This choice allows: (i) to avoid prohibitive filling times due to significant differences of size and (ii) to get a sufficient fine representation of both the media (fiber and matrix) and the interface.

At this stage, being able to represent damage mechanisms at the fiber scale does not present any interest except for the final failure but this will be useful in the future when intra tow fissuring will be considered.

\subsection{Representative Elementary Volume (REV)}

A cubic domain is considered as the Representative Elementary Volume (REV). It is made of a cylindrical carbon fiber flooded in an epoxy matrix. Results are widely available in the literature [24] for such a REV. It also corresponds to a will to avoid too prohibitive times of simulations. The main objective at this stage is to prove the interest of the present DEM for the modeling of the damage mechanisms in composite. In parallel to this work, one can precise developments are performed in the laboratory to model cells containing several fibers randomly distributed within a Statistical Elementary Volume (SEV) such as those considered in [25]. The way of parallel computing is explored.

For the present REV, the fiber is assumed to be of a cylindrical shape. Its diameter is such as the volume rate of fiber is of the order of $51.3 \%$. This corresponds to an arbitrary fixed value. The length of the cell in the fiber direction results from an analysis of sensibility [25]. The Fig. 1(a) presents the elementary volume by distinguishing the cylindrical volume of the fiber of that cubic of the matrix.

\subsection{DEM modeling of the REV: the filling technic}

The filling of the REV by the discrete elements has to enable a correct representation of the continuums (fiber and matrix). The filling operation is challenged by the following objectives: (i) to reach a rate of compaction for modeling correctly the continuums [26], (ii) to insure the media isotropy [26] (the carbon fiber anisotropy is not yet considered but envisaged in future works), (iii) to preserve a definition of the geometry in the interface fiber/matrix as precise as possible.

The most effective one consists into two steps. The first stage consists in filling the whole volume of the cubic cell by aiming at the rate of compaction of reference of 6.3 [26] without distinction of the volumes of the fiber and the matrix. This filling makes in two operations:

- A first operation of random trial in the space of the possible positions is realized insuring the geometrical strict condition of not overlapping of DE.

- The second operation consists in introducing a set of DE by forcing their overlapping in the restricted volume of REV. DEM calculations are then performed to release internal energy until obtaining the desired rate of compression.

The second stage simply consists in differentiating DE belonging to the fiber of those belonging to the matrix, so as to obtain the volume rate of fiber wished (desired), that is $51.3 \%$ (Fig. 1(b)). The distinction of fiber DE of those of the matrix is simply made by locating the position of the DE centers close to the interface represented by a perfect cylindrical envelope. So, the centers of DE located in the envelope will be allocated to the fiber whereas those situated out- 


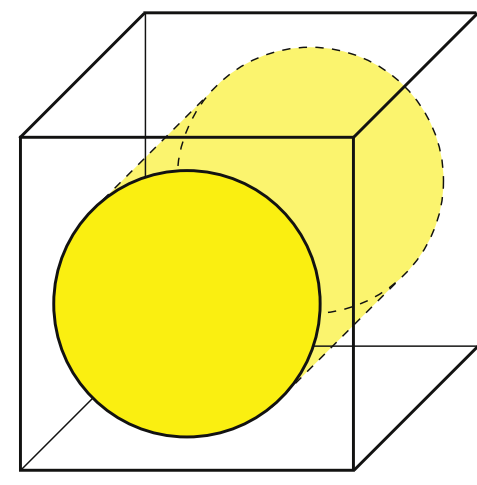

(a)

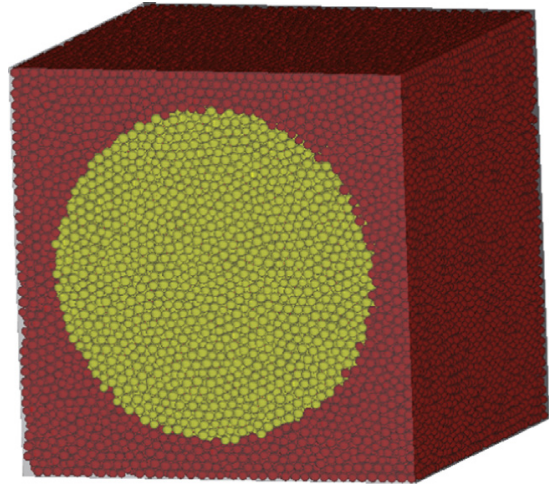

(b)

Fig. 1. REV definition. (a) Geometry. (b) Discretization with 80,000 DE.

side will be associated to the matrix. In this way, the cross section profile of the interface is not a perfect circle, Fig. 1(b). In the reality, the fibers are not being perfectly cylindrical, so this geometrical modeling can turn out relevant. The numbers of DEs used in the model in Fig. 1(b) are: 41,731 the fiber, 38,749 for the matrix, so 80,480 in all. The radius of DEs varies according to a uniform distribution so as to insure the isotropy of the domain [26]. The sample is a cube of $8.66 \mu \mathrm{m}$ edge.

\section{Mechanical modeling}

\subsection{General considerations}

From the geometry, it is now necessary to place the links between the fiber DEs and the matrix DEs but also between the fiber/matrix DEs in order to model the behavior of each continuum and at the interface. For the REV in Fig. 1(b), 130,516 fiber links, 115,096 matrix links and 8282 fiber-matrix links were generated between DEs. The mechanical behavior of these links is assumed to be brittle elastic.

Whereas many authors [12-14,16,17] use contact and spring links between particles in DE simulations, authors of the present study prefer use cohesive beams developed in [26,27] and no contact model. These links introduced at the microscopic scale are calibrated to find the elastic and failure properties observable at a superior scale. Continuum fiber and matrix, and interface properties are addressed. For the moment, the links are identical for a given medium but a natural variability is envisaged in the future by simply varying the properties of these links.

In this study, the UD composite defined in Section 2.3 has material properties extracted from literature [28]. They are listed in Table 1 . Thereafter, the subscripts ${ }_{M}$ and ${ }_{\mu}$ denote respectively the Macroscopic and microscopic variables whereas the superscripts ${ }^{f i b}$, mat and ${ }^{f-m}$ denote respectively a variable concerning the fiber, the matrix and the fiber-matrix.

$\mathrm{E}_{M}, \rho_{M}, v_{M}, \sigma_{\text {fail }}$ are respectively the macroscopic Young modulus, the density, the Poisson ratio, the macroscopic stress failure and $\mathrm{D}$, the fiber diameter. Hereafter, isotropic properties are adopted for the carbon fiber by convenience. This assumption has no consequence for primary studies presented here. The transverse isotropy of the carbon fiber will be introduced in future works.

\subsection{Calibration procedure}

\subsubsection{Elastic behavior}

The elastic behavior of the cohesive beam bond is defined by four parameters: two geometrical ones, the length $l_{\mu}$ and the radius
Table 1

Calibration of the discrete bounds properties of fiber and matrix.

\begin{tabular}{|c|c|c|c|c|c|}
\hline & $\rho\left[\mathrm{kg} \mathrm{m}^{-3}\right]$ & $E$ [GPa] & $v$ & $r$ & $\sigma_{\text {fail }}[\mathrm{GPa}]$ \\
\hline \multicolumn{6}{|l|}{ Carbone fiber } \\
\hline Continuum properties $_{M}$ & 1750 & 260 & 0.3 & - & 2.5 \\
\hline Discrete bounds properties $\mu$ & & 8272 & 0.3 & 0.307 & \\
\hline with the BBF process & & & & & 86.2 \\
\hline with the RDEF process & & & & & 5.45 \\
\hline \multicolumn{6}{|l|}{ Epoxyde matrix } \\
\hline Continuum properties ${ }_{M}$ & 1200 & 3.45 & 0.3 & - & 0.07 \\
\hline Discrete bounds properties $\mu$ & & 89 & 0.3 & 0.33 & \\
\hline with the BBF process & & & & & 2.0 \\
\hline with the RDEF process & & & & & 0.148 \\
\hline
\end{tabular}

$R_{\mu}$, and two mechanical ones, the Young modulus $E_{\mu}$ and the Poisson's ratio $v_{\mu}$. The bond length $l_{\mu}$ is the distance between two DE centers. It is automatically constrained by the filling procedure. Instead of using the beam radius $R_{\mu}$, the adimensional beam radius $r_{\mu}=R_{D E} / R_{\mu}$ is preferred, where $R_{D E}$ is the mean radius of all the spherical DE. This parameter and the two others have to be determined by a calibration procedure. As shown and performed in [26], the calibration is based on numerical tensile tests on cylindrical beams using about $10000 \mathrm{DE}$. The procedure developed by [26] is used for the parameter determination and the results are resumed in Table 1.

\subsubsection{Fragile behavior}

Two failure criteria have been investigated in this work in order to appreciate the relevance of each other in modeling the failure process. The first one is the most employed in the literature $[8,29,30]$ whereas the second one has been developed in the lab $[31,32]$. The comparison between the two approaches is looked for at this stage.

The first criterion is the 'breakable bonds failure process', $B B F$, is driven by the failure of the bonds when a tensile criterion is satisfied inside the bond $b$. This tensile criterion is based on the maximum normal stress and simply stipulates: failure if $\sigma_{b \mu}>\sigma_{\text {fail } \mu}$ and no failure if not. Then, $\sigma_{\text {fail }}$ depending on the nature of the bond, i.e. the fiber, the matrix or the fiber-matrix bonds, the calibration procedure is used to get it.

The second criterion is the 'removed DE failure process', $R D E F$, is based on the deletion of a DE when a tensile criterion is satisfied in bonds connected to this DE. A virial tensor is defined for each DE $i$ as following:

$\bar{\sigma}_{i}=\frac{1}{2 \Omega_{i}} \sum_{i \neq j} \frac{1}{2}\left(r_{i j} \otimes f_{i j}+f_{i j} \otimes r_{i j}\right)$ 
Each virial tensor $\bar{\sigma}_{i}$ is a function of the forces and the moments, $f_{i j}$, of the bonds connecting the DE $i$ to the DE $j$ spaced of $r_{i j}$. This criterion is the one used by [32] and needs a calibration procedure to be identified.

The fragile calibration procedure is then performed with the addition of another step, the determination of $\sigma_{\text {fail }}$ (BBF and RDEF processes). Calculations are performed for several values of $\sigma_{\text {fail }}$. The microscopic stress failure $\sigma_{\text {fail }}$ is determined by matching the looked value with the expected macroscopic stress failure $\sigma_{\text {failm }}$.

\subsection{About the fiber-matrix links}

As a first approximation, the fiber-matrix links are kept identical to those of the links matrix. Adhesive contact laws building is now in progress in the laboratory and will be soon substituted for these links. The assumption is sufficient for present objective. So, only conclusions on the model relevance to capture cracks mechanisms can be dressed. No conclusions on the model accuracy to predict any physical damage mechanisms can be established yet.

\section{Elementary tests on a UD composite cell}

The elementary tests aim at validating the homogenized properties obtained for the composite using the present DEM for both elastic and rupture behavior. This is the first step of this work before using such a model to locally capture damage mechanisms directly coupled with the global structure, or with a more industrial vision, to return degraded homogenized properties issued from DEM to the global structure [5]. A cubic cell (Fig. 2(a)) of size $a=1=8.66 \mu \mathrm{m}$ with a fiber of diameter $\phi=7 \mu \mathrm{m}$ is used for these elementary tests.

The development and the implementation of particular programs (or plugins) within the platform GranOO of the laboratory [21] allow to illustrate the broken links (in the matrix, in the fibers as well as in the fiber/matrix interface) according to the load. For each test, kinematic boundary conditions are adopted by convenience of implementation but force, mixed or periodic boundary conditions remain completely possible.

From the numerical simulations, the relevance of the model and its capacity to report mechanisms of degradation at the RVE microscopic scale can be appreciated both qualitatively and quantitatively by visualizing the break of the links.

\subsection{The performed tests}

Four first numerical tests have been performed on the elementary cell (Fig. 2(a)) in order to obtain the elastic properties of the anisotropic homogeneous media and to compare them with the literature. The failure process is therefore non-actived for these four tests.

- A tensile test, referenced by $\# \mathrm{~L}$, is performed to determine the elastic modulus $E_{L}$ along the fiber direction, Fig. 3(a). The face $x=0$ is prevented from any $x$-displacement. A $x$-displacement is imposed on the opposite face $x=l$, Fig. 2(b).

- A tensile test, referenced by \#T, is performed to get the elastic modulus $\mathrm{E}_{T}$ along the direction orthogonal to the fiber, Fig. 3(b). The face $y=0$ is prevented from any $y$-displacement. A $y$-displacement is imposed on the opposite face $y=a$, Fig. 2(b).

- A shear test, referenced by \#LT, is realized to obtain the shear modulus $\mathrm{G}_{L T}$ in $L T$ plane, Fig. 3(c). The face $z=0$ is prevented from any displacement. A $x$-displacement is imposed on the opposite face $z=a$, Fig. 2(b).

- A shear test, referenced by \#TT, is finally performed to determine the shear modulus $G_{T T^{\prime}}$ in $T T^{\prime}$ plane, Fig. 3(d). The face $z=0$ is prevented from any displacement. A $y$-displacement is imposed on the opposite face $z=a$, Fig. 2(b).

The numerical tests have been performed with several discrete domains built as described in Section 2. The characteristics of the studied discrete domains are resumed in Table 2 . The influence of the number of DE and the influence of the random position of the DE have been studied. A volumic fraction of fiber arbitrary chosen at $51.3 \%$ is retained for simulations.

A numerical sensor located at the loading face for each test has been used to measure the evolution of the force in order to calculate a macroscopic stress in the elementary cell. The present DEM is naturally formulated in an explicit time integration scheme adapted to model dynamic problem, impacts on composite being aimed later. The time step is about $10^{-9} \mathrm{~s}$. No numerical or physical damping is introduced. So, non-stabilized oscillations subsist in the following curves and originate in round trip of waves in the elementary cell.

In this validation step, the viscous behavior of the epoxy matrix is first neglected. Only a brittle elastic behavior is taken into account.

Table 3 summarizes both the numerical results obtained with present method and analytical ones issued from literature $[33,34,28]$.

\subsection{Quantitative results on elastic properties}

Elastic properties such as elastic moduli (Fig. 4) and Poisson's ratios (Fig. 5) can be obtained with the two first elementary tests whereas shear moduli (Fig. 6) can be assessed with the two last elementary tests.

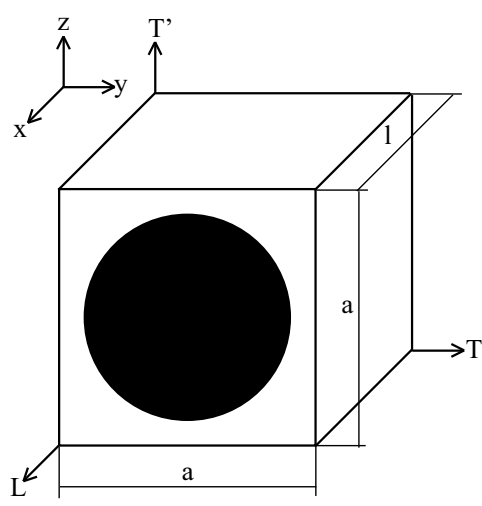

(a)

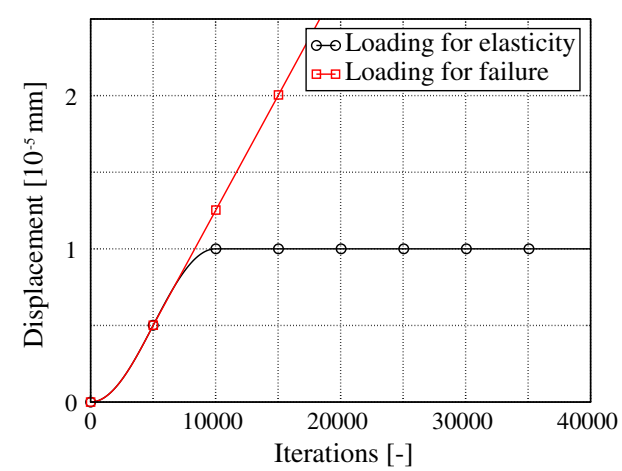

(b)

Fig. 2. (a) Elementary cell. (b) Displacement loadings. 


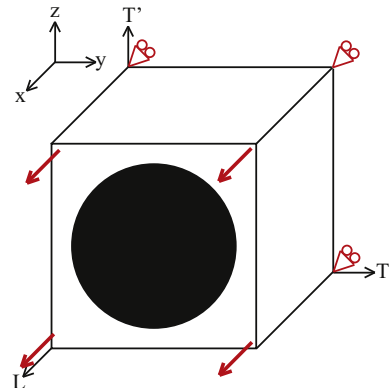

(a)

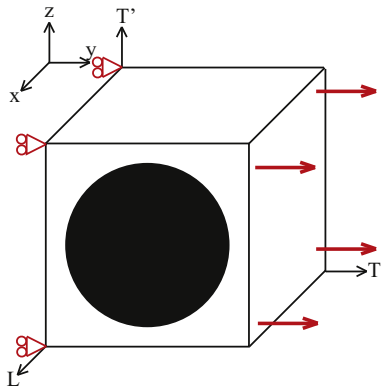

(b)

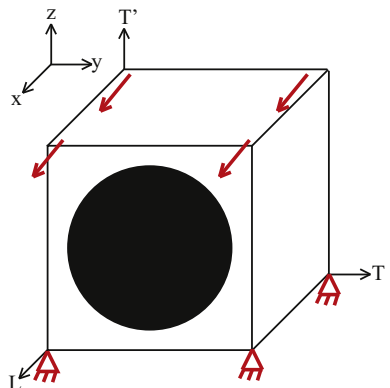

(c)

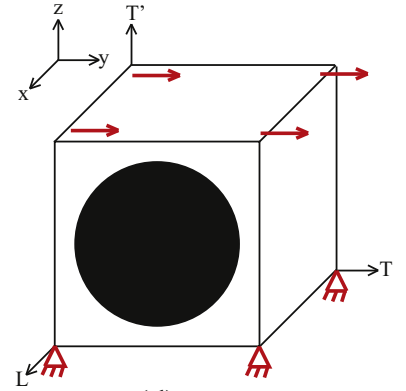

(d)

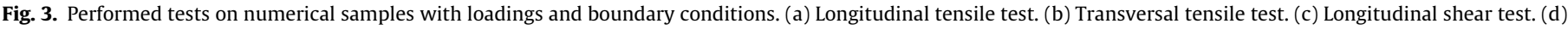
Transversal shear test.

Table 2

Characteristics of numerical samples.

\begin{tabular}{|c|c|c|c|c|c|c|c|}
\hline \multirow[t]{2}{*}{ Sample } & \multicolumn{3}{|c|}{$\mathrm{Nb}$ of $\mathrm{DE}$} & \multicolumn{3}{|c|}{$\mathrm{Nb}$ of bounds } & \multirow[t]{2}{*}{ F-M } \\
\hline & Total & Fiber & Matrix & Total & Fiber & Matrix & \\
\hline$\# 1$ & 40,730 & 21,149 & 19,581 & 131,375 & 67,504 & 58,468 & 5403 \\
\hline$\# 2 a$ & 60,297 & 31,292 & 29,005 & 189,578 & 97,561 & 85,288 & 6729 \\
\hline$\# 2 b$ & 62,001 & 32,098 & 29,903 & 200,071 & 101,824 & 91,238 & 7009 \\
\hline$\# 2 c$ & 62,305 & 32,212 & 29,918 & 200,897 & 102,805 & 90,979 & 7113 \\
\hline$\# 3$ & 80,480 & 41,731 & 38,749 & 253,894 & 130,516 & 115,096 & 8282 \\
\hline
\end{tabular}

Table 3

Elastic and failure properties of the UD composite obtained with the theory, the DE method and the FE method.

\begin{tabular}{|c|c|c|c|c|c|c|c|c|c|c|}
\hline \multicolumn{2}{|l|}{ Sample } & $E_{L}[\mathrm{GPa}]$ & & $E_{T}[\mathrm{GPa}]$ & \multicolumn{2}{|c|}{$v_{L T}$} & $v_{T T^{\prime}}$ & \multicolumn{2}{|l|}{$G_{L T}[\mathrm{GPa}]$} & $G_{T T^{\prime}}[\mathrm{GPa}]$ \\
\hline \multicolumn{11}{|c|}{ Elastic properties } \\
\hline \multicolumn{2}{|c|}{ Theory [28] } & 135.06 & & 9.52 & \multicolumn{2}{|c|}{0.3} & 0.3 & \multicolumn{2}{|l|}{3.98} & 3.41 \\
\hline \multicolumn{2}{|c|}{ \#1 } & 153 & & 12.5 & \multicolumn{2}{|c|}{0.290} & 0.288 & 2.74 & \multicolumn{2}{|c|}{2.58} \\
\hline \multicolumn{2}{|l|}{$\# 2 \mathrm{a}$} & 139 & & 11.5 & \multicolumn{2}{|c|}{0.296} & 0.303 & 2.43 & \multicolumn{2}{|c|}{2.36} \\
\hline \multicolumn{2}{|l|}{$\# 2 b$} & 151 & & 12.9 & \multicolumn{2}{|c|}{0.290} & 0.290 & 2.54 & \multicolumn{2}{|c|}{2.57} \\
\hline \multicolumn{2}{|l|}{$\# 2 c$} & 154 & & 12.8 & & & 0.290 & 2.69 & & \\
\hline \#3 & & 140 & & 11.5 & & & 0.301 & 2.42 & & \\
\hline FEM dy & licit & 134 & & 10.7 & - & & - & 2.33 & & \\
\hline FEM qu & & 134 & & 10.7 & 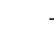 & & - & 2.32 & & \\
\hline Sample & $\sigma_{\text {failM }}$ & & $\varepsilon_{\text {failm }}[$ & & $\%$ of & onds & & & & \\
\hline & & & & & Fibe & & Mat & & Fibe & \\
\hline & BBF & RDEF & $\mathrm{BBF}$ & RDEF & BBF & RDEF & BBF & RDEF & BBF & RDEF \\
\hline Failure 1 & & & & & & & & & & \\
\hline$\# 1$ & 1.42 & 1.45 & 10.00 & 10.20 & 4.6 & 7.1 & 44 & 25 & 99 & 40 \\
\hline$\# 2 \mathrm{a}$ & 1.30 & 1.38 & 9.97 & 10.90 & 4.5 & 7.0 & 42 & 22 & 93 & 38 \\
\hline$\# 2 \mathrm{~b}$ & 1.30 & 1.40 & 8.86 & 10.10 & 3.7 & 6.5 & 45 & 17 & 98 & 28 \\
\hline$\# 2 c$ & 1.36 & 1.44 & 9.16 & 9.80 & 4.1 & 6.5 & 40 & 19 & 99 & 30 \\
\hline \#3 & 1.34 & 1.34 & 9.69 & 10.35 & 4.1 & 6.2 & 48 & 27 & 91 & 39 \\
\hline
\end{tabular}

\subsubsection{Comparison of elastic and shear moduli}

The value of numerical moduli $E_{L}, E_{T}, G_{L T}$ and $G_{T T^{\prime}}$ and the value of numerical Poisson's ratios $v_{L T}$ and $v_{T T^{\prime}}$ have been approached by a sliding average value calculated over several time periods excluding the 10,000 first iterations of the loading ramp. Results are presented in Table 3.

Firstly, the elastic moduli are overestimated by numerical simulations compared to reference values whereas the shear moduli are underestimated. However, numerical results are in the same range with the theoretical ones. Same remark can be done for Poisson's ratios; suitable values are obtained in the same range than the theoretical ones. These results confirm a good tendency. They could be significantly improved using more matrix DEs in the narrowest area of $(a-D) / 2$ size to reduce edge effects.

Secondly, it can be observed that elastic and shear moduli are not influenced by the number of $\mathrm{DE}$ but more by the procedure to build discrete samples. The filling procedure to build discrete domains can therefore influence the elastic properties and show the interest of the DEM to build domains including a natural variability.

\subsubsection{Origin of the observed oscillations}

Oscillations can be observed on all the plotted figures (see Figs. 4-6) and originate in wave propagation and reflection inside the numerical sample. The dynamic property of the calculation involves such oscillations. Finite Element (FE) simulations have also been performed with the software code Herezh++ [35] using a dynamic explicit time integration scheme such as in [36] or a quasi-static one. The FE mesh presented in Fig. 7(a) is composed of 32,067 nodes and 58,320 linear pentahedron. The four elementary tests have been performed using the FE description with the explicit time integration scheme in order to exhibit the oscillations 


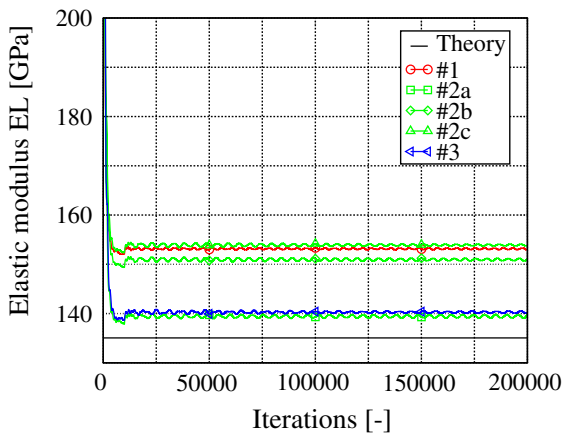

(a)

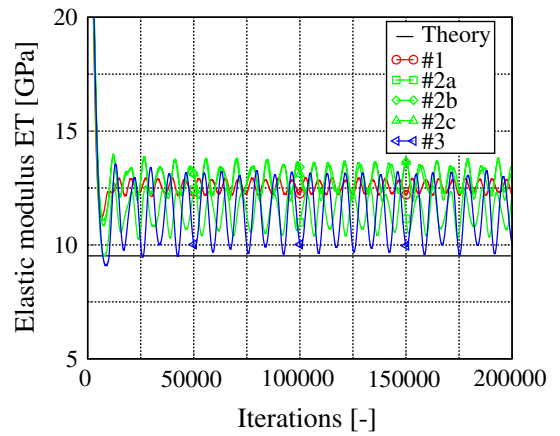

(b)

Fig. 4. Determination of elastic moduli with elementary tests on the UD composite cell. (a) Determination of $E_{L}$. (b) Determination of $E_{T}$.

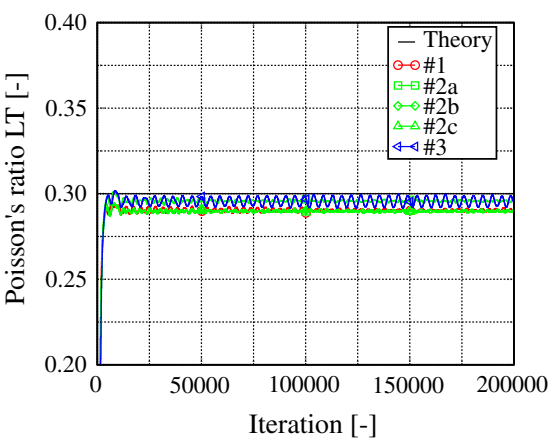

(a)

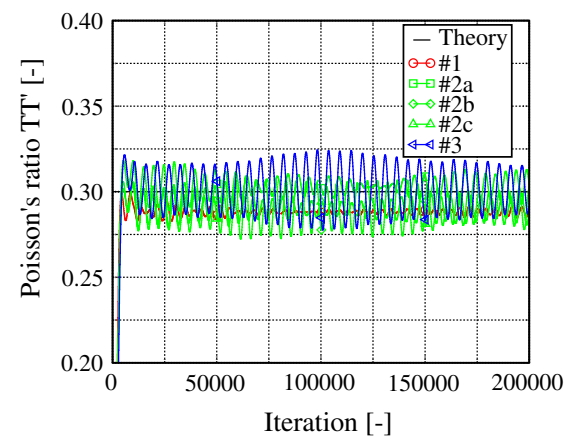

(b)

Fig. 5. Determination of Poisson's ratios with elementary tests on the UD composite cell. (a) Determination of $v_{L T}$. (b) Determination of $v_{T T^{\prime}}$.

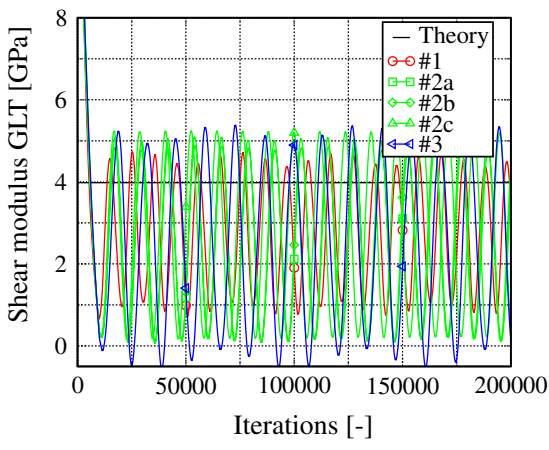

(a)

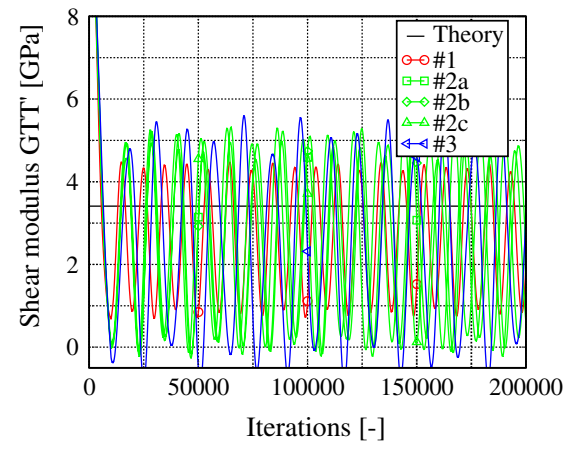

(b)

Fig. 6. Determination of elastic moduli with elementary tests on the UD composite cell. (a) Determination of $G_{L T}$. (b) Determination of $G_{T T^{\prime}}$.

phenomenon. The same oscillations with the same periods and amplitudes than with the DE simulations can be observed. The evolution of the $G_{T T^{\prime}}$ is only presented here (Fig. 7(b), black curve). A sliding average (Fig. 7(b), red curve ${ }^{1}$ ) has been calculated over the stabilized duration, i.e. $\left[10^{-5}: 10^{-4}\right] \mathrm{ms}$, with the same manner than for the DE results. A FEM quasi-static has also been performed to get the stabilized response of the numerical sample (Fig. 7(b), green curve). The sliding average method seems therefore to be a good tool to determine the quasi-static stabilized response. FE results are resumed and can therefore be compared with DE ones and theoretical ones in Table 3.

\footnotetext{
${ }^{1}$ For interpretation of color in Fig. 7, the reader is referred to the web version of this article.
}

\subsection{Qualitative results about rupture behavior}

The elementary cell is now subjected to the L-tensile test previously described with a linear displacement loading applied until the failure, red curve of Fig. 2(b). The theoretical macroscopic failure strain values for the fiber and the matrix are respectively $\varepsilon_{\text {failm }}^{\text {fib }}=9.6 \cdot 10^{-3}$ and $\varepsilon_{\text {fail }}^{\text {mat }}=20.3 \cdot 10^{-3}$. The two failure criterion $B B F$ and $R D E F$ using a stress formulation are presented above and are evaluated in the present section.

\subsubsection{Breakable Bonds Failure process (BBF)}

Fig. 8 presents the results obtained with the \#2b sample. The stress-strain curve is plotted and the evolution of the broken beams, i.e. the fiber, the matrix and the fiber-matrix materials links, is superposed in Fig. 8(a). A zoom of the failure stage is plot- 


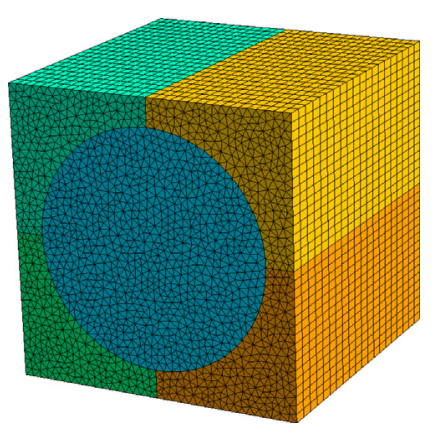

(a)

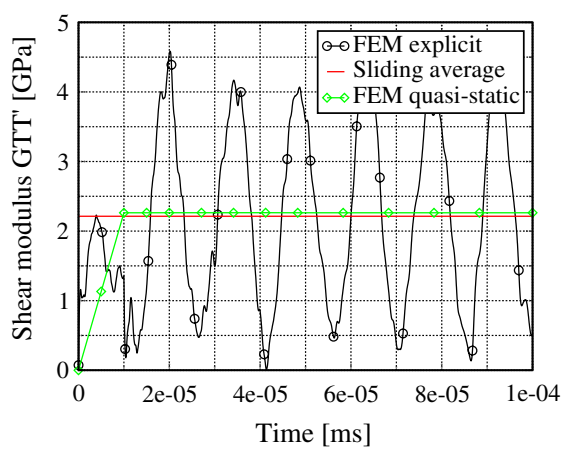

(b)

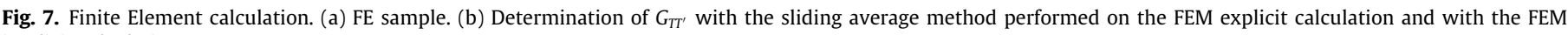
implicit calculation.

ted in Fig. 8(b) with four relevant times (a)-(d). The face and the profile views of the sample can be observed in Fig. 9 to illustrate the propagation of the failure inside the elementary cell. The fiber-matrix bonds are only drawn in red in order to make the figures understandable and broken bonds are drawn in blue. A transparency displaying is chosen and every bonds are therefore displayed through the sample. As already mentioned, these results have the sole purpose to validate the use of the failure criterion and then, to show the DEM ability to represent damage kinetic until the rupture. Indeed, take an interest in a fiber damage mechanisms is quite questionable but according to the authors, this fiber will be advantageously replaced by a tow in future investigations. Nevertheless, four main stages can just be described and commented:

(a) During a first large macroscopic 'elastic-damaged' stage $\left(\varepsilon_{M} \in\left[0: 8.82 \cdot 10^{-3}\right]\right)$, a few number of broken bonds randomly appears in the fiber. It can be noticed that the broken bonds tend to progressively appear in a place located at the bottom center of the fiber (see Fig. 9(a) and (e)).

(b) During a second 'fiber crack' stage $\left(\varepsilon_{M} \in\left[8.82 \cdot 10^{-3}: 9.00\right.\right.$ $\left.\left.10^{-3}\right]\right)$, the crack propagates from the place where the broken bonds are gathered through the entire section of fiber (see Fig. 9(b) and (f)). The stress level drops (see Fig. 8) while the number of fiber broken bonds increases until to a maximum of almost $4 \%$ of the total fiber bonds (see Fig. 8). At the same time, the number of fiber-matrix broken bonds hugely increases while the matrix beams just begin to break.

(c) During a third 'propagation in the fiber-matrix interface' stage $\left(\varepsilon_{M} \in\left[9.00 \cdot 10^{-3}: 9.25 \cdot 10^{-3}\right]\right)$, the crack mainly propagates through the interface between fiber and matrix (see Fig. 9(c) and (g)) which is corroborated by the number of fiber-matrix broken bonds which reaches $90 \%$ of the total fiber-matrix bonds (see Fig. 8).

(d) During a forth stage $\left(\varepsilon_{M} \approx 9.5 \cdot 10^{-3}\right)$, the crack goes on mainly propagating through the interface between fiber and matrix and more slightly through the matrix (see Fig. 9(d) and (h)). Indeed, there are almost no broken bounds in the corners of the discrete domain while their concentration in the fiber-matrix interface is important (see Fig. 9(h)). The number of fiber-matrix and matrix broken bonds finally respectively reaches a maximum of $98 \%$ and $45 \%$ for a macroscopic strain of $16.00 \cdot 10^{-3}$ (see Fig. 8) and show that the degradation of the discrete domain mainly occurs in the interface and cannot be neglected inside the matrix.

This failure test has been repeated for the other referenced samples and shows the same failure procedure as described previously, i.e. (i) initiation of the crack inside the fiber, (ii) propagation through the fiber, (iii) propagation at the interface fiber-matrix and (iv) degradation of the discrete domain mainly in this interface area. The main results of these numerical tests are resumed in Table 3. Even if these results are only qualitatively analyzed for now, they show the DEM ability to represent damage kinetic until the rupture.

\subsubsection{Removed Discrete Element Failure process (RDEF)}

The RDEF process is performed for each $D E$ of the discrete domain. A virial stress tensor is calculated by the mean of forces and torques in neighbor bonds as described in Section 3.2.2. When this virial stress reaches the microscopic failure limit, i.e. $\sigma_{\text {fail } \mu}^{\text {fib }}=5450 \mathrm{MPa}$

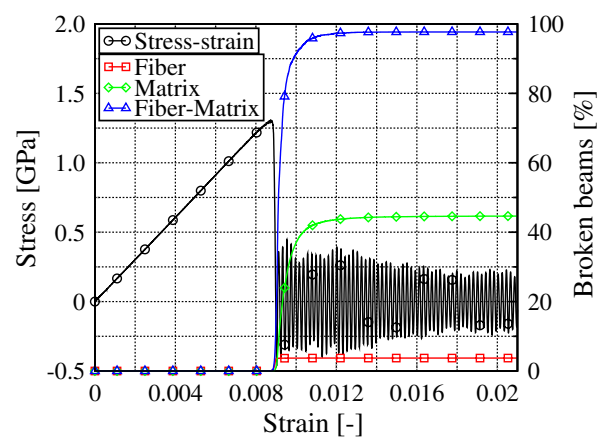

(a)

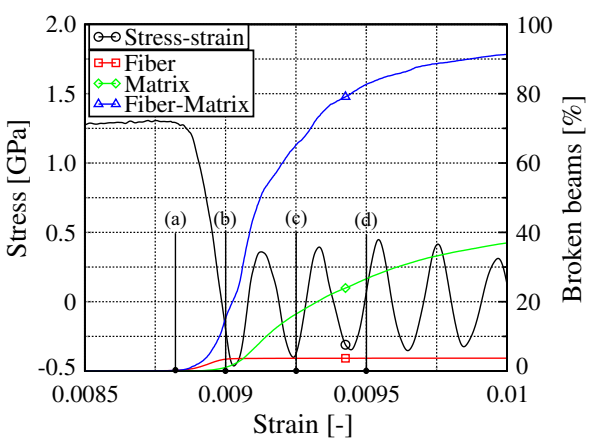

(b)

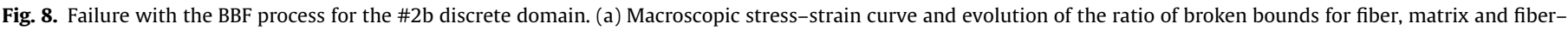
matrix bonds. (b) Zoom during the failure. 


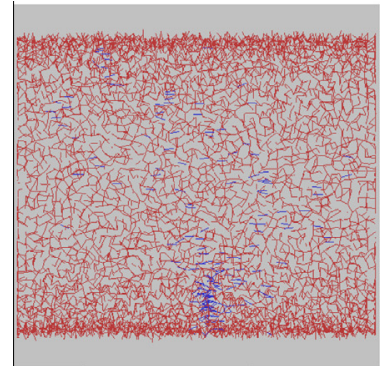

(a)

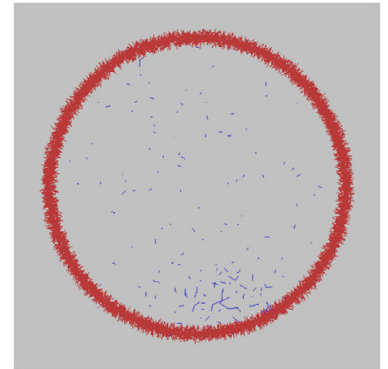

(e)

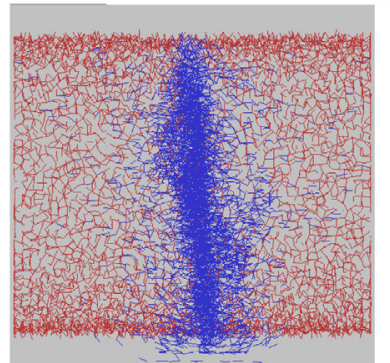

(b)

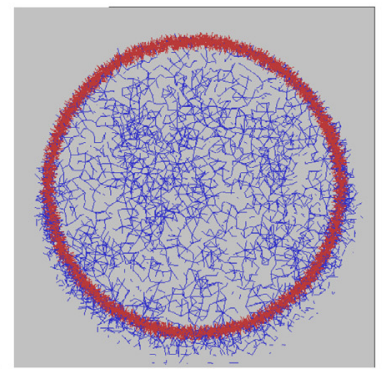

(f)

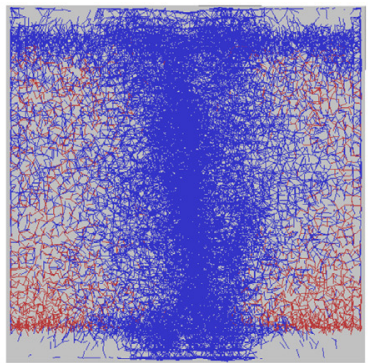

(c)

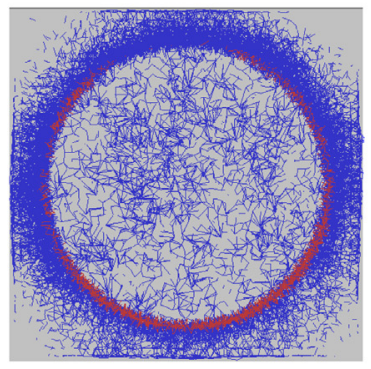

(g)

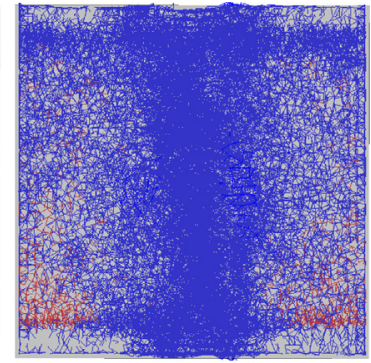

(d)

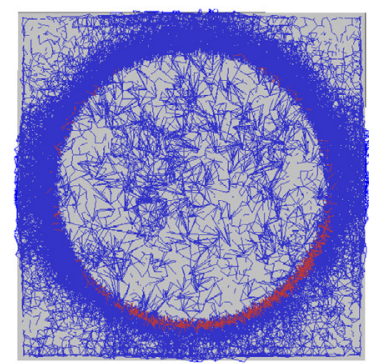

(h)

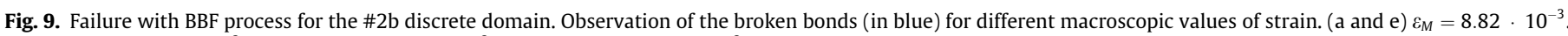

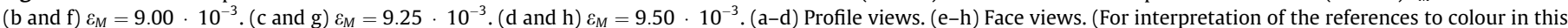
figure legend, the reader is referred to the web version of this article.)

and $\sigma_{\text {fail }}^{\text {mat }}=148 \mathrm{MPa}$ for the fiber and the matrix respectively, the DE is deleted and therefore all the neighbor bonds are also deleted. In this study, the DE is deleted but furtherly, this DE will be kept in order to model debris and the contact between this DE and the adjacent will be treated. In order to make understandable the comparison between the two failure processes, the deleted bonds will be displayed in the following figures.

With the same manner than previously, four main stages can be described and analyzed:

(a) During a first large macroscopic 'elastic-damaged' stage $\left(\varepsilon_{M} \in\left[0: 10.00 \cdot 10^{-3}\right]\right)$, a few number of broken bonds randomly appears in the fiber (see Fig. 11(a) and (e)). It can be noticed that damages appear progressively in the fiber during a longer strain duration than with the previous process (see Fig. 10(a) and (b)). Another difference between the two failure processes comes from the place where the crack initiates even if the discrete domains are the same.

(b) During a second 'fiber crack' stage $\left(\varepsilon_{M} \in\left[10.00 \cdot 10^{-3}\right.\right.$ : $\left.10.25 \cdot 10^{-3}\right]$ ), the crack propagates from the place where the broken bonds are gathered through the entire section of fiber (see Fig. 11(b) and (f)). The stress level drops while the number of fiber broken bonds increases until to a maximum of almost $6.5 \%$ of the total fiber bonds (see Fig. 10). At the same time, the number of matrix and fiber-matrix broken bonds hugely increases. It can be noticed that the macroscopic strain $\varepsilon_{\text {fail }}$ of the fiber crack is higher with the RDEF process than with BBF one, $10^{-4}$ and $9 \cdot 10^{-3}$, respectively.

(c) During a third 'propagation in the matrix' stage $\left(\varepsilon_{M} \in\right.$ $\left.\left[10.25 \cdot 10^{-3}: 10.50 \cdot 10^{-3}\right]\right)$, the crack mainly propagates through the matrix (see Fig. 11(c) and (g)) which is corroborated by the number of matrix broken bonds which reaches $17 \%$ of the total matrix bonds (see Fig. 10). This stage is completely different with this process than with the previous one. Indeed, with the RDEF process, the crack propagates through the matrix following the crack path initiated inside the fiber. (d) During a forth stage $\left(\varepsilon_{M} \approx 12.00 \cdot 10^{-3}\right)$, the crack keeps mainly propagating through the matrix and more slightly through the fiber-matrix interface (see Fig. 11(d) and (h)). Indeed, we can be notice the presence of broken bounds in the corners of the discrete domain which shows the entire failure of the numerical specimen (see Fig. 11(h)). The number of fiber-matrix and matrix broken bonds finally respectively reaches a maximum of $28 \%$ and $17 \%$ for a macroscopic strain of $12.00 \cdot 10^{-3}$ (see Fig. 10). These values show that the degradation does not occur in the entire specimen but only in the failure area.

\section{Conclusion and future works}

The first works on 3D modeling of an UD composite material cell by using the Discrete Elements Method (DEM) developed in the laboratory are presented in this paper. The composite material is restricted to a cubic Representative Elementary Volume (REV) constituted by a carbon fiber flooded in an epoxy matrix. The geometrical modeling of this elementary volume with discrete elements of spherical shape is first treated by distinguishing the fiber of the matrix. The filling phase is described in particular to get a good representativeness of the continuity of each isotropic medium (fiber and matrix) by respecting a rate of compaction and an optimal number of coordinations.

The mechanical modeling of the fiber and the matrix as continuous media but also the interface fiber-matrix is then considered. It consists of the introduction of mechanical links intended to model the constitutive behavior. At this stage of the works, the objective is mainly to prove the relevance of the present 3D DEM for the representation of the damage mechanisms in a UD composite cell. So, a simple brittle elastic behavior is adopted for the fiber and for the matrix to prove this feasibility. The thermal, plastic, viscous aspects already investigated in $[37,38]$ could be later implemented if needed by the physics. These mechanical links are beam model whose the properties are calibrated to find the macroscopic mechanical properties. At the interface, the links between 


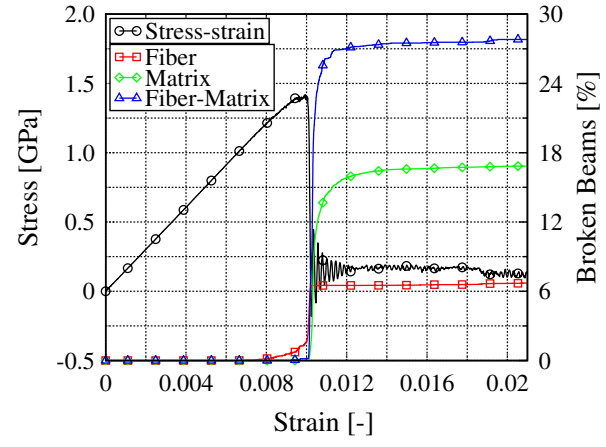

(a)

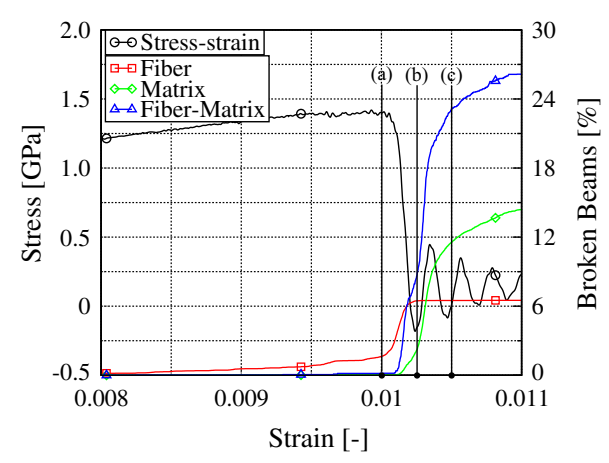

(b)

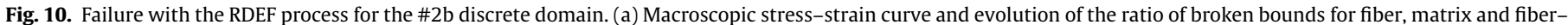
matrix bonds. (b) Zoom during the failure.

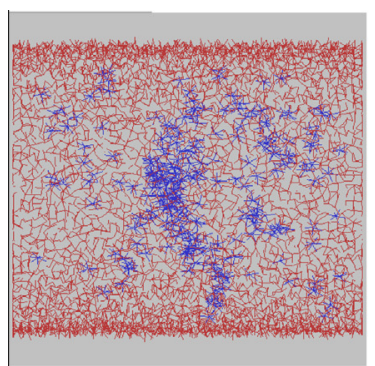

(a)

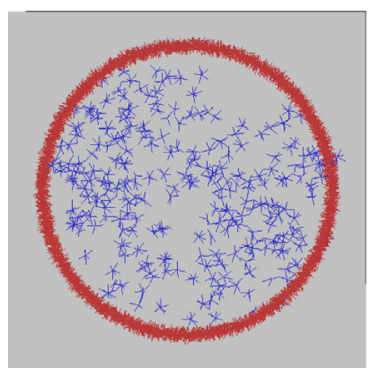

(e)

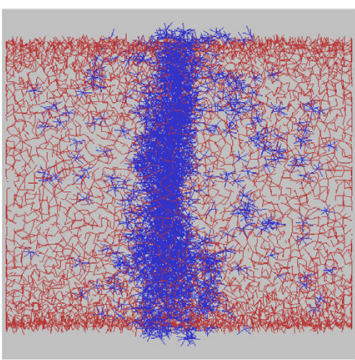

(b)

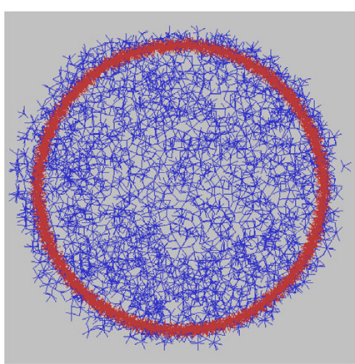

(f)

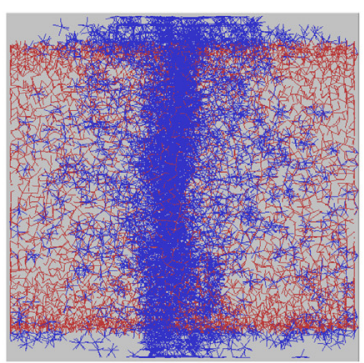

(c)

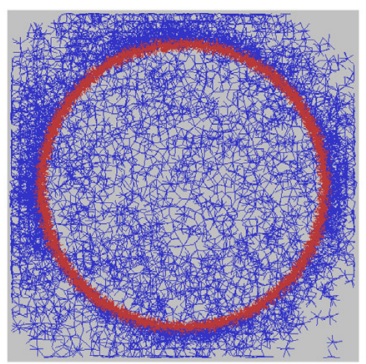

(g)

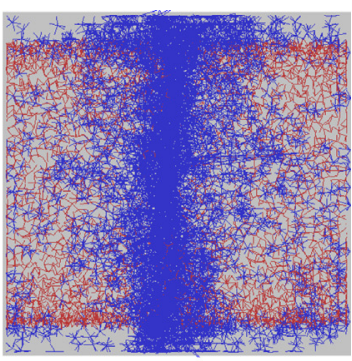

(d)

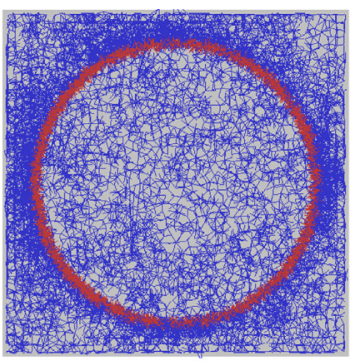

(h)

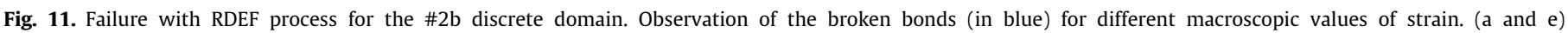

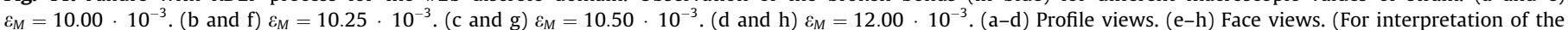
references to colour in this figure legend, the reader is referred to the web version of this article.)

the fiber and matrix are identical to the links of the matrix at this stage.

Once the geometrical and mechanical modelings accomplished, elementary tests on the REV are performed. They allow to estimate the anisotropic properties of the REV and to appreciate the capabilities to capture the damage mechanisms with the present DEM. The cases of longitudinal and transversal tractions, in plane and out of plane shearing are performed.

In spite of interface links largely improvable, the results obtained on the anisotropic elastic properties from the REV are nevertheless in the good order of height of those issued from analytical models taken as reference. The longitudinal $E_{L}$ and transversal $E_{T}$ Young modulus are overestimated whereas the $G_{L T}$ and $G_{T T}$ shear modulus are underestimated.

Besides, the two criteria implemented to assess the DEM capabilities in representing the damage mechanisms influence the damage progress. A more localized rupture zone is observed using the Breakable Bonds Failure criterion compared with the Removed DE Failure one. The pictures plotted for different REV strain state show the present DEM ability to model the kinetics of the damage. Globally, the results encourage the authors to go on in this way.

So, numerical investigations are already in progress to implement a more physical contact at the interface using cohesive laws. The double cantilevered beam (damage in mode 1 ), shearing tests (damage in modes 2 and 3 ) and tests combining damage in mode 1 and mode 2 (or mode 3 ) are experienced before validating the approach on a pull-out test. Once acquired the damage mechanisms modeling, passing from the REV to a Statical Elementary Volume (SEV) is the next step. The interest will be to represent the damage mechanisms when several fibers are included in a matrix volume and then to be able to directly couple such local effects to the global ones by sticking method [9] or to indirectly couple 
them returning degraded properties from the SEV modeled with the present DEM to the global structural scale modeled with FEM or equivalent method.

\section{References}

[1] Kelley FS. Mesh requirements for the analysis of a stress concentration by the specified boundary displacement method. In: ASME, proceedings of the second computers in engineering international conference; 1982.

[2] Ransom JB, McCleary SL, Aminpour MA, Knight Jr NF. Computational methods for global/local analysis. Technical Memorandum 107591, NASA; 1992.

[3] Mao KM, Sun CT. A refined global-local finite element analysis method. Int J Numer Methods Eng 1991;32:29-43.

[4] Whitcomb JD. Iterative global/local finite element analysis. Comput Struct 1991;40(4):1027-31.

[5] Terada K, Kikuchi N. A class of general algorithms for multiscale analyses of heterogeneous media. Comput Methods Appl Mech Eng 2001;190:5427-64.

[6] Song J-H, Belytschko T. Multiscale aggregating discontinuities method for micromacro failure of composites. Compos Part B: Eng 2009;40(6):417-26.

[7] Cundall PA, Strack ODL. A discrete numerical model for granular assemblies. Géotechnique 1979;29:47-265.

[8] Kun F, Herrmann HJ. A study of fragmentation processes using a discrete element method. Comput Methods Appl Mech Eng 1996;138(1-4):3-18.

[9] Jebahi M, Charles JL, Dau F, Illoul L, Iordanoff I. 3D coupling approach between discrete and continuum models for dynamic simulations (DEM-CNEM). Comput Methods Appl Mech Eng 2012;255:169-209.

[10] Dhia HB, Rateau G. The Arlequin method as a flexible engineering design tool. Int J Numer Methods Eng 2005;62(11):1442-62.

[11] Jebahi M, Dau F, Charles JL, Iordanoff I. Simulation of laser-induced damage in fused silica using the DEM-CNEM coupling method; 2013 [in preparation].

[12] Wittel FK, Schulte-Fischedick J, Kun F, Kröplin B-H, Frieb M. Discrete element simulation of transverse cracking during the pyrolysis of carbon fibre reinforced plastics to carbon/carbon composites. Comput Mater Sci 2003;28: $1-15$.

[13] Wittel FK, Kun F, Kröplin B-H, Herrmann HJ. A study of transverse ply cracking using a discrete element method. Comput Mater Sci 2003;28(3-4):608-19.

[14] Sheng Y, Yang D, Tan Y, Ye J. Microstructure effects on transverse cracking in composite laminae by DEM. Compos Sci Technol 2010;70:2093-101.

[15] Biscaia HC, Micaelo R, Teixeira J, Chastre C. Numerical analysis of FRP anchorage zones with variable width. Composites Part B 2014;67:410-26.

[16] Yang D, Sheng Y, Ye J, Tan Y. Discrete element modeling of the microbond test of fiber reinforced composite. Comput Mater Sci 2010;49:253-9.

[17] Yang X, Xia Y, Zhou Q. Influence of stress softening on energy-absorption capability of polymeric foams. Mater Des 2011;32(3):1167-76.

[18] Iordanoff I, Battentier A, Néauport J, Charles JL. A discrete element model to investigate sub-surface damage due to surface polishing. Tribol Int 2008; 41(11):957-64.

[19] Shiu W, Donzé FV, Daudeville L. Penetration prediction of missiles with different nose shapes by the discrete element numerical approach. Comput Struct 2008;86(21-22):2079-86.
20] Aghazadeh Mohandesi J, Refahi A, Sadeghi Meresht E, Berenji S. Effect of temperature and particle weight fraction on mechanical and micromechanical properties of sand-polyethylene terephthalate composites: a laboratory and discrete element method study. Composites Part B 2011;42(6):1461-7.

[21] André D, Charles JL, Iordanoff I, Terreros I. GranOO, a discrete workbench; 2013. <http://www.granoo.org>.

[22] Voronoi G. Nouvelles applications des paramètres continus à la théorie des formes quadratiques. Reine Angew Math 1907;133:97-178.

[23] Yvonnet J, Ryckelynk D, Lorong P, Chinesta P. Interpolation naturelle sur les domaines non convexes par l'utilisation du diagramme de Voronoi contraintMéthode des éléments C-Naturels. Rev Eur élém finis 2003;12(4):487-509.

[24] Sun CT, Vaidya RS. Prediction of composite properties from a representative volume element. Compos Sci Technol 1996;56:171-9.

[25] Raducu Chermaneanu, Représentation de la variabilité des propriétés mécaniques d'un CMO à l'échelle microscopique: Méthodes de construction des distributions statistiques. PhD Thesis, Université de Bordeaux I, France; 2012.

[26] André D, Iordanoff I, Charles J-L, Néauport J. Discrete element method to simulate continuous material by using the cohesive beam model. Comput Methods Appl Mech Eng 2012;213-216:113-25.

[27] André D. Modélisation par éléments discrets des phases d' ébauchage et de doucissage de la silice. PhD Thesis, Université de Bordeaux I, France; 2012.

[28] Berthelot J-M. Matériaux Composites - Comportement mécanique des structures. 2nd Ed. Paris: Masson; 1996.

[29] Camborde F, Mariotti C, Donzé FV. Numerical study of rock and concrete behaviour by discrete element modeling. Comput Geotech 2000;27(4): 225-47.

[30] D'Addetta GA, Kun F, Ramm E. On the application of a discrete model to the fracture process of cohesive granular materials. Granul Matter 2002;4(2): 77-90.

[31] Jebahi M, André D, Dau F, Charles JL, Iordanoff I. Simulation of Vickers indentation of silica glass. J Non Cryst Sol 2013;378:15-24.

[32] André D, Jebahi M, Iordanoff I, Charles J-L, Néauport J. Using the discrete element method to simulate brittle fracture in the indentation of a silica glass with a blunt indenter. Comput Methods Appl Mech Eng 2013;265:136-47.

[33] Hashin Z. On elastic behaviour of fibre reinforced materials of arbitrary transverse phase geometry. J Mech Phys Solids 1965;13:119-34.

[34] Hill R. Theory of mechanical properties of fibre-strengthened materials: I. Elastic behavior. J Mech Phys Solids 1964;12:199-212.

[35] Rio G. Herezh++: FEM software for large transformations in solids, Dépôt APP certification IDDN.FR.010.0106078.000.R.P.2006.035.20600. Laboratoire d'ingénierie des matériaux de Bretagne; 2013.

[36] Maheo L, Grolleau V, Rio G. Numerical damping of spurious oscillations: a comparison between the bulk viscosity method and the explicit dissipative Tchamwa-Wielgosz scheme. Comput Mech 2013;51(1):109-28.

[37] Goupil A. Modélisation du lissage de défauts sur les optiques asphériques de photolithographie: approche par éléments discrets. PhD Thesis, Arts et Metiers Paristech, France; 2013.

[38] Terreros I. Modélisation DEM thermo-mécanique du milieu continu. Vers la simulation du procédé FSW. PhD Thesis, Arts et Metiers Paristech, France; 2013. 